\section{(6) OPEN ACCESS}

\title{
Work-related road traffic injury: a multilevel systems protocol
}

\author{
Sharon Newnam, ${ }^{1}$ Dianne M Sheppard, ${ }^{1}$ Mark A Griffin, ${ }^{2}$ Roderick J McClure, \\ Gillian Heller, ${ }^{3}$ Malcolm R Sim, ${ }_{1}^{4}$ Mark R Stevenson ${ }^{1}$
}

${ }^{1}$ Accident Research Centre, Monash Injury Research Institute, Monash University, Melbourne, Australia ${ }^{2}$ School of Psychology, The University of Western Australia, Perth, Western Australia, Australia

${ }^{3}$ Department of Statistics, Macquarie University, Sydney, New South Wales, Australia ${ }^{4}$ Monash Centre for Occupational \& Environmental Health (MonCOEH), School of Public Health \& Preventive Medicine, Monash University, Melbourne, Victoria, Australia

\section{Correspondence to} Dr Sharon Newnam Monash Injury Research Institute, Building 70, Clayton Campus, Monash University, VIC 3800, Australia; sharon.newnam@monash.edu

Received 16 December 2013 Revised 2 January 2014 Accepted 8 January 2014 Published Online First 29 January 2014
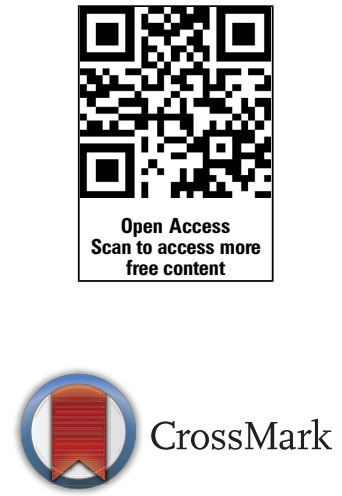

To cite: Newnam $S$, Sheppard DM, Griffin MA, et al. Inj Prev 2014;20:e6.

\begin{abstract}
Background Although road traffic injury is reported as the leading cause of work-related death in Australia, it is not clear, due to limitations in previous methods used, just how large a burden it is. Many organisations are unaware of the extent of work-related road traffic injury and, importantly, what can be done to reduce the burden. The proposed research will (i) estimate the prevalence of work-related road traffic injury and (ii) identify the organisational determinants associated with work-related road traffic injury.

Methods and design The current study is designed to enumerate the problem and identify the individual driver-level, the supervisor-level and organisational-level factors associated with work-related road traffic injury. The multilevel systems protocol will involve a series of cross-sectional surveys administered to drivers of fleet vehicles $(n=1200)$, supervisors of the drivers $(n=1200)$ and senior managers $(n=300)$ within the same
\end{abstract} organisation.

Discussion The novel use of the multilevel systems protocol is critical to be able to accurately assess the specific determinants of driving safety within each context of an organisation.

Results The results are expected to highlight that reducing injury in the workplace requires more than just individual compliance with safety procedures. It will also establish, for the first time, an occupational translation taskforce to ensure that the research findings are adopted into work-place practice and thereby directly contribute to reductions in work-related road traffic injury.

\section{BACKGROUND}

More than $30 \%$ of registered motor vehicles in Australia are work-related vehicles, with an estimated $33 \%$ of work-related fatalities occurring while driving. ${ }^{1}$ In the state of New South Wales (NSW) alone, fleet vehicles (defined as vehicles that have a business registration or vehicles with a private registration but where the registered operator is an organisation $)^{2}$ comprise up to 5.3 fatalities per 100000 registered fleet vehicles, ${ }^{3}$ and there is evidence suggesting an over-representation of work-related drivers in road traffic injury compared with non-work-related drivers. ${ }^{4-7}$ This emerging public health problem is not solely occurring in Australia, with work-related road traffic deaths estimated to account for $22 \%$ of work fatalities in the USA and $16 \%$ in New Zealand. ${ }^{1}$

\section{Enumerating work-related road traffic injury}

Traditionally, work-related road traffic injury has been enumerated through worker's compensation databases. However, these estimates are unlikely to represent the true extent of the problem due to the differences in state-based compensation schemes. Furthermore, these systems rely on labour force data from all industries and occupations, regardless of whether the individuals within those sectors use vehicles in the course of their work. Thus, worker's compensation databases are likely to under-represent the prevalence of work-related road traffic injuries.

Alternative methods of enumerating work-related road traffic injury, including registration-based data $^{38}$ and self-reported data, ${ }^{9} 10$ are also limited. In regards to the former, estimation through registration-based data is not feasible as only one state jurisdiction in Australia (NSW) has mandatory work-use vehicle registration. In regards to the latter, there is considerable opportunity for false reporting, particularly when the road injury may be linked with unsafe work practices. Given the problems associated with existing methodologies, an alternative method of estimating the magnitude of work-related road traffic injury is required. For many jurisdictions, the magnitude of work-related road traffic injury can only be estimated within organisations. Proxy measures of work-related road traffic injury including lost time injury (LTI), as defined under the Worksafe Australian National Standard, ${ }^{11}$ would provide an ideal basis upon which to calculate the rate of work-related road traffic injury. Thus, the first aim of this study is to enumerate work-related road traffic injury using LTI.

\section{Identifying the organisational determinants of work-related road traffic injury}

In the field of work-related road traffic injury prevention, organisational factors are receiving increasing attention in terms of their role in the safety of fleet vehicle drivers. Past research has identified factors such as a driver's perception of the value given to safety in the organisation, ${ }^{7} 12$ the driver's daily workload, ${ }^{4}{ }^{14} 15$ attitudes to safety expressed by the driver's supervisor ${ }^{16}$ and their own driving practices ${ }^{17}$ as predictors of safety outcomes. However, these studies have been conducted primarily within a single organisation, and therefore they have not been able to isolate the key organisational factors that predict differences between organisations in outcomes such as workrelated road traffic injuries.

Better understanding of organisational determinants is critical to our ability to reduce workrelated road traffic injury. Much has been learned in recent years about the organisational differences 
that influence safety behaviours. For example, the quality of management practices within organisations has been linked to reduced injury rates ${ }^{18}$; however, these findings have not been tested in relation to reduced work-related road traffic injury.

To achieve reductions in work-related road traffic injury, it will be necessary to focus beyond the individual's compliance with safety procedures. ${ }^{19}$ A safe work environment also depends on individuals anticipating threats to safety, showing concern for the safety of others and contributing to safety improvements in the organisation. These behaviours are strongly influenced by the system of interlinked contexts operating at multiple levels within the organisation. Figure 1 provides an overview of the determinants of work-related road traffic injury within the multilevel contexts that shape and constrain these determinants. The proposed project will incorporate, in a novel and unique way, important features of the context at each level and assess specific determinants of driving safety within each context.

At the broader organisational level, the context includes the business activities that could predispose drivers to more unsafe driving conditions. For example, it is possible that individuals within organisations in which driving is the core business (eg, transport ancillaries) are exposed to better safety cultures compared with organisations where driving is secondary to the individual's core role responsibility (eg, in-home nursing care). The key determinant of work-related road traffic injury that this study will consider is the nature of high-performance workplace systems $\left(\mathrm{HPWS}^{20}\right.$ ).

HPWS are distinct but interconnected human resource management practices that are designed to improve workplace competence, attitudes and motivation. Of importance, there is a strong association between HPWS and occupational safety, ${ }^{18}$ which highlights that reducing injury in the workplace requires more than just individual compliance with safety procedures.

At the supervisor or team level, the context is characterised by the social interaction among team members and the interdependence among tasks. The way supervisors manage these features of the context is an important determinant of safety behaviour within the team. Safety practices of supervisors such as monitoring, goal setting and feedback will be important to assess as these factors have been shown to have a strong impact on safety within teams, ${ }^{21}$ thus making interdependence among tasks an important feature of the context at this level of a driver's experience. The management of safety in the workrelated driving setting has some characteristics that distinguish it from the management of other organisational safety activities.

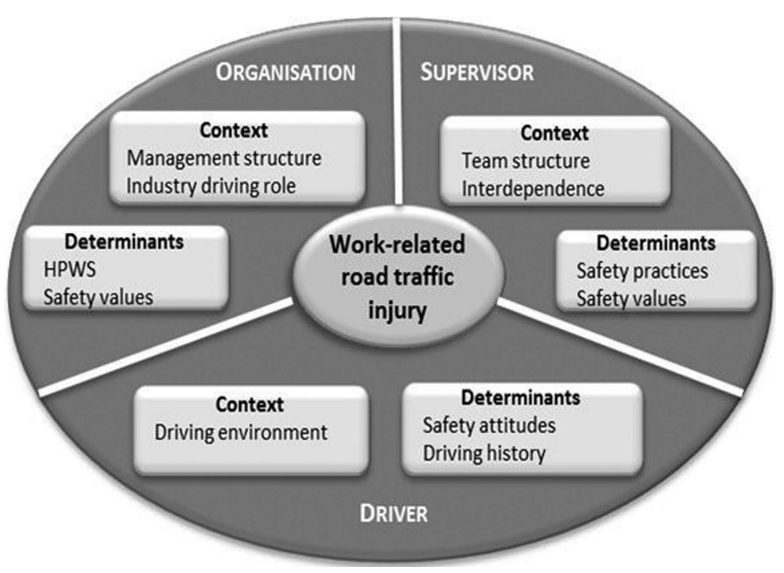

Figure 1 The context and determinants of work-related road traffic injury.
Driving activities often fall outside typical line management responsibilities and are supervised by a person who is not part of the same management structure associated with other work roles (ie, a fleet manager). Therefore, the allocation and management of supervisory responsibilities for safe driving will also be important to assess within the group.

At the individual level, the specific driving experiences of individual drivers will be incorporated, along with motivational determinants of safety such as safety attitudes and driving history. A comprehensive assessment of individual-level variables will allow us to control for the multiple causes of road traffic injury that exist beyond the organisation. Overall, these three levels of analysis will provide a comprehensive picture of the various organisational, team and individual factors that impact on individual driving safety.

In summary, despite the statistics highlighting the overrepresentation of work-related road traffic fatalities, there is limited understanding of the magnitude of work-related road traffic injury. The proposed research will apply novel methods that will take account of these limitations and provide, for the first time, an estimate of the prevalence of work-related road injury. As well, the proposed research will consider the role of organisational determinants of work-related road traffic injury. To determine how these processes influence the likelihood of work-related road traffic injury, this study will investigate how key factors influence the propensity for injury at multiple levels within the same organisation. Consequently, the unique findings from this research, when implemented, will have direct relevance to a large proportion of the Australian workforce.

\section{METHODS/DESIGN}

\section{Study design and setting}

The multilevel systems protocol will involve a series of three cross-sectional surveys administered to drivers of fleet vehicles, supervisors of the drivers and senior managers within the same organisation. Subsequent multilevel modelling, when applied, will identify the significant organisational determinants associated with work-related road traffic injury in organisations. To obtain a sample of fleet vehicle drivers, the investigators will be facilitated by a leading fleet vehicle insurer (insuring 50\% of all fleet vehicles in the states of NSW and Victoria (VIC)) who will provide the introduction to organisations who have fleet vehicles insured with them.

\section{Sample size and recruitment}

Following the organisations' agreement to participate (potentially 1181 organisations), a two-stage sampling approach will be undertaken. The first stage will involve a stratified random sample of 300 organisations. To ensure a balanced representation between large organisations (100 or more fleet vehicles) and small-to-medium-sized organisations (10-99 fleet vehicles), small-to-medium-sized organisations will be sampled at a higher rate (approximately 30\% higher). Each organisation will be asked to identify a senior manager (defined as a person that has administrative or managerial authority in the organisation), and the research team will approach the manager to seek their participation in the study. A total of 300 senior managers will ensure we have sufficient power $(80 \%)$ to detect a significant $(p<0.05)$ organisation-level factor to be associated with a work-related road traffic injury. The recruitment approach described here for senior managers has been trialled in previous research by the investigators with response rates of senior managers of $64 \%{ }^{9}$; hence, 472 organisations will initially be approached. 
The second stage will involve a random sample of fleet vehicle drivers from the selected organisations. Each organisation will provide a de-identified listing of all fleet vehicle drivers who were employed with the organisation between 1 January 2011 and 31 December 2012. Based on the data of the fleet vehicle insurer as at 31 December 2011, the sampling frame will comprise 3000 fleet vehicle drivers. A total of 1200 fleet drivers will be required in order to have sufficient power to detect a significant difference in the incidence of work-related traffic injury between two groups, with a power of 0.80 . Based on previous research with this target population, ${ }^{9}$ a response fraction of $56-60 \%$ is anticipated; hence, a random sample of 2150 fleet vehicle drivers will be invited (via their organisation) to participate in the study. Finally, the participating fleet vehicle drivers will be asked to identify their relevant supervisor or team leader (see definition below). We will use email links to invite supervisors to participate in the study. This process will allow us to match individual-level and supervisor-level responses in a multilevel structure. This methodology has been previously used by the investigators with a response rate of $53 \% .^{9}$ Consequently, we anticipate a sample of 400 supervisors.

\section{Data collection and measurement}

The fleet vehicle drivers will participate in a structured telephone interview using a computer-assisted telephone interviewing system (CATI system). Prior to the telephone interview, all telephone interviewers will undergo training on the instrument to be administered to each driver to ensure a standard protocol is adhered to. Ten per cent of the telephone interviews will be supervised, and a further $10 \%$ of the telephone interviews will be validated by a follow-up phone call by a second interviewer. We will employ the Western Australian Survey Research Centre at Edith Cowan University to undertake the interviews.

The interview will take approximately $20 \mathrm{~min}$ to administer, and the structured interview will elicit driver demographics, tenure in current role and organisation, driving experience, estimate of the kilometres travelled over the observational period and involvement in a work-related road traffic crash during the observational period, and if so, whether the crash resulted in an injury requiring loss time at work of more than 1 day following the crash. For the purposes of this research, a work-related road traffic crash resulting in lost time at work of more than 1 day will be deemed a work-related road traffic injury. This concurs with a LTI standard defined under Worksafe Australia National Standard. ${ }^{11}$ The LTI self-report data will also be verified by the human resource department within each organisation to assess the validity of reporting; this will be undertaken across a $10 \%$ random sample of respondents. The interview will also involve a series of questions that relate to a driver's attitudes to safety, including perceptions of safety at the workgroup and organisational levels, attitudes towards driving safely, self-efficacy and work overload. We will use the three-item group-level safety values ${ }^{22}$ and the nine-item organisational-level safety values (communication, inspections, training ${ }^{19}$ ). Self-efficacy will be assessed with a three-item scale, ${ }^{23}$ and attitudes towards driving safely will be measured by an 11-item scale. ${ }^{24}$ All of these scales have been used by the coauthors and modified to suit the workrelated driving context. ${ }^{9} 14$ To assess driving behaviour, we will use the Occupational Driver Behaviour Questionnaire. ${ }^{14}$
At the supervisor level, we will obtain data on the supervisory practices that support or hinder safe driving. Supervisors play an important role in establishing the importance of safety in the local work environment through monitoring safety requirements, establishing goals for safety and providing feedback about achieving the set goals. ${ }^{25} 26$ Furthermore, supervisors have been found to create the context in which safety is valued by drivers and other safety representatives (ie, fleet managers) in the organisation. ${ }^{9}$ The data will be obtained via an online survey that will be distributed to the driver's direct supervisor (defined as those who monitor and regulate drivers in their performance of assigned tasks, both driving and non-driving related). The contact details of the supervisors will be provided by the fleet vehicle drivers after they have completed their telephone survey. An email will then be sent to the supervisor outlining the purpose of the study, inviting them to participate and providing a link to the online survey. This approach has been used in past research conducted by the coauthors with considerable success. ${ }^{9}$ The survey will assess safety practices using Zohar and Luria's ${ }^{27}$ group-level safety climate scale. This employee-oriented scale will be modified to directly assess supervisors' perceptions of the extent to which they engage in the key content themes: active practices (monitoring, controlling), proactive practices (instructing, guiding) and declarative practices (declaring, informing). We will also assess the value given to safety by supervisors using a three-item safety values scale. $^{22}$

At the organisation level, the data will be obtained through a $30 \mathrm{~min}$ telephone interview with senior managers. The telephone interviews will be conducted by trained research staff appointed on the project in each of the respective states. We seek information from each manager regarding whether their organisation has systems and practices that support an organisation's safety culture. In particular, we will adopt the perspective of HPWS, which measures resource-oriented practices, such as communication, training, selection and job design. There is a strong association between HPWS and occupational safety, ${ }^{18}$ highlighting that reducing injury in the workplace requires more than just individual compliance with safety procedures. HPWS will be assessed using a high-performance management practices scale, designed for this study and informed by a conceptual framework developed by Posthuma et al. ${ }^{28}$ We will also assess the value given to safety by senior-level management using the safety values scale. ${ }^{22}$

\section{Statistical analysis}

Two statistical approaches will be applied to address the proposed aims. The first, using the individual-level data from the random sample of fleet vehicle drivers, we will estimate the prevalence of work-related road traffic injury, while the second will consider the nested structure of the data (namely, drivers, supervisors and managers within the organisation) in order to identify the organisational determinants associated with workrelated road traffic injury.

The statistical approach to estimating the prevalence of workrelated road traffic injuries (as defined by vehicle fleets, namely commercial vehicles less than 4.5 tonnes) in the states of NSW and VIC will be calculated by weighting the responses obtained by the inverse of their probability of selection as determined by (i) the probability of selecting the organisation, (ii) the probability of selecting a male or female driver and (iii) the number of drivers in the organisation. 
Different weights will be calculated for the organisations and the drivers. First, the weight for organisation i will be generated as

$$
\mathrm{H}_{\mathrm{ik}}=\frac{1}{\mathrm{P}_{\mathrm{ik}}} \times \frac{1}{\mathrm{t}_{\mathrm{i}}}
$$

where $t_{i}$ defines the number of drivers in the organisation and $\mathrm{P}_{\mathrm{ik}}$ the probability of selecting the ith driver. The weight for the drivers $k$ of gender $\mathrm{j}$ will be given by

$$
\mathrm{W}_{\mathrm{ijk}}=\mathrm{H}_{\mathrm{ik}} \frac{1}{\mathrm{P}_{\mathrm{ij}}} \times \mathrm{n}_{\mathrm{ij}}
$$

where $P_{i j}$ reflects the probability of selecting the jth gender in the ith organisation and $n_{i j}$ will be the combination of organisation and gender of the driver. Consequently, the sample weight $\mathrm{R}_{\text {iik }}$ that will be applicable to denote the probability that driver $\mathrm{k}$ of gender $\mathrm{j}$ from the organisation $\mathrm{i}$ being selected for the survey is given by

$$
\mathrm{R}_{\mathrm{ijk}}=\mathrm{W}_{\mathrm{ijk}} \times \frac{1}{\mathrm{r}_{\mathrm{ijk}}}
$$

To take account of the bias due to non-response of drivers and to ensure the sample reflects the NSW and VIC population distribution of insured fleet vehicles, a fourth weighting will be applied that takes into consideration the distribution of all fleet vehicles of the five companies who insure the remaining $50 \%$ of fleet vehicles in the two states. ${ }^{29}$

All data obtained from the structured interviews will be analysed and prevalence rates and associated 95\% CIs will be computed using standard formulae for calculating prevalence rates. ${ }^{30}$ The rates will be adjusted for the effects of intraclass correlation associated with the organisation. Random effects models will be used to estimate this effect, and if significant, estimates of the SEs of the prevalence rates will be appropriately adjusted for it.

Multilevel modelling will be applied to identify the significant organisational determinants associated with work-related road traffic injury in organisations. At the individual driver level, relevant covariates (see data collection and measurement) will be adjusted for and a correction for exposure (driving kilometres) applied. At the organisation level, occupational groupings along with other covariates will be investigated and included in the model (if found to be a significant predictor at the 5\% significance level). Models for the total number of work-related road traffic injuries will be formulated based on a Poisson or negative binomial response distribution as appropriate. Random effects will be used to model within-organisation correlation in the generalised linear mixed models framework. ${ }^{31}$ Such models will be fitted using the statistical software SAS (proc glimmix). The resultant model will identify the variables associated with the risk of work-related road traffic injury at the individual driver, team supervisor and organisation levels. An understanding will also be obtained in relation to the variation of injury rates across drivers 'nested within' organisations.

\section{DISCUSSION}

Many organisations are unaware of the extent of the problem of work-related road traffic injury or the factors within their organisations that are likely to influence potential reductions in work-related road traffic injury and deaths. This study will not only enumerate the problem but it will identify the driver-level, supervisor-level and organisational-level factors associated with work-related road traffic injury.

To date, the magnitude of work-related road traffic injury is hidden within the overall burden of road injury. There are many reasons for this, including (i) a lack of uniformity across statutory data sources, (ii) limitations in the methods used to estimate work-related road traffic injury and (iii) the fact that there are few proxy measures for estimating the extent of the problem. The proposed research will apply novel methods that will take account of these limitations and provide, for the first time, an estimate of the magnitude of work-related road injury.

Understanding of the organisational determinants is critical to our ability to reduce work-related road traffic injury. Although much has been learned about the organisational differences that influence safety behaviours, the findings have not been investigated in relation to work-related road traffic injury. To achieve reductions in work-related road traffic injury, it will be necessary to extend the focus beyond the individual's compliance with safety procedures, as safe work environments depend on individuals anticipating threats to safety, showing concern for the safety of others (team supervisor) and contributing to safety improvements in the organisation. These behaviours are strongly influenced by the organisational context. The proposed study will be the first to consider the organisational context in relation to work-related road traffic injury and take account of the complex system when identifying its organisational determinants.

Importantly, until now, almost all research related to organisations and work-related road traffic injury has been undertaken within single organisations. A limitation of these studies is that it is unknown whether the key safety factors identified within each organisation generalise to other organisations, particularly organisations with differing business activity and size. Furthermore, it is unknown whether the business activities operating within particular organisations or industries predispose drivers to unsafe driving conditions. Thus, this research will not only examine the organisational determinants of work-related road traffic injury but importantly take account of the core business activity of the organisations.

With almost one-third of registered motor vehicles in Australia used for work purposes, the implications of the primary aim of this study, identifying organisational determinants associated with work-related road traffic injury, are substantial. Interventions arising from the findings of this research will contribute significantly to reductions in work-related road traffic injury and will support the National Road Safety Strategy target of a $30 \%$ reduction in serious injuries by $2030 .^{32}$

\section{Practical applications}

Enhancing the interface between research and policy and practice is crucial, and we will promote our research findings by facilitating the links between the employers (organisations), employees (drivers), as well as regulatory policymakers and researchers. In this project, we will establish an Occupational Translation Task Group (OTTG) that will facilitate interaction in relation to the research findings. The OTTG will have representatives from the insurance industry, government departments, health and road agencies along with representation from two large and small-to-medium-sized vehicle fleet operators. It will also be important that employees injured in work-related driving accidents are represented. The OTTG will focus on facilitating an interaction between all of the aforementioned bodies and will be the main facilitator for translating the 
research findings into organisational practice and other outcomes.

\section{Considerations}

The authors acknowledge that there are limitations to inferring the causal direction from a cross-sectional study. The authors spent considerable time deliberating over the choice of the proposed research design. Cross-sectional design was selected over a longitudinal design for two reasons. First, given that we are at the early stages of understanding the theoretical and empirical foundations associated with organisations, their management and the behaviour of staff, we deemed that it would be a prohibitively expensive and risky exercise to pursue a longitudinal design. Second, research by one of the coauthors has shown that the climate created by supervisors exerts a strong impact on individual safety performance, with positive effects on safety performance with lags of up to 2 years. ${ }^{22}$ Consequently, given the timeframe being examined in the proposed study, there is every likelihood that if the supervisory environment has not changed in the $12-24$ months prior to the event then there is the potential for a causal direction to be inferred.

In regards to generalisability, the authors are confident that a random sample from the participating insurance database (stratified by the vehicle fleet size) will ensure that findings can be generalised to fleet drivers in the states of VIC and NSW. The participating insurance agency is the largest fleet insurer in the two states with 50\% coverage (a further five insurers cover the remainder of vehicle fleets in both states). The insurance agency has 1118 policies, which include small, medium and large fleet sizes and have both private and public (government) fleet vehicle policies across a range of industry groups (eg, agricultural/farming, manufacturing, charity organisations, hospitals). Importantly, it is unlikely that the remaining fleet insurers in VIC and NSW would differ in the fleet size insured.

\section{SUMMARY}

Road traffic injury is the leading cause of work-related death in Australia. ${ }^{33}$ Despite this, many organisations are unaware of the extent of the problem or the factors within their organisations that are likely to influence potential reductions in work-related road traffic injury and deaths. This study will not only enumerate the problem and identify the individual driver-level, supervisor-level and organisational-level factors associated with work-related road traffic injury, it will establish, for the first time, an occupational translation taskforce to ensure the research findings are adopted into work-place practice and thereby directly contribute to reductions in work-related road traffic injury.

Contributors SN and MRS conceptualised the program of research. SN, MRS, MAG, GH, RJM and MRS all contributed to writing the grant application. DMS is the manager for this 3 -year program of research. All authors contributed to writing this manuscript.

Funding The research is funded by Australia's National Health and Medical Research Council (NHMRC) Project Grant scheme (APP1041754).

Competing interests MRS is supported by a research fellowship from the NHMRC, Australia.

Patient consent Obtained.

Ethics approval This project was approved by the Monash University Human Research Ethics Committee (MUHREC) on 14/3/2013 (CF12/4063-2012001963).

Provenance and peer review Not commissioned; externally peer reviewed.

Open Access This is an Open Access article distributed in accordance with the Creative Commons Attribution Non Commercial (CC BY-NC 3.0) license, which permits others to distribute, remix, adapt, build upon this work non-commercially, and license their derivative works on different terms, provided the original work is properly cited and the use is non-commercial. See: http://creativecommons.org/ licenses/by-nc/3.0/

\section{REFERENCES}

1 Driscoll T, Marsh S, McNoe B, et al. Comparison of fatalities from work related motor vehicle traffic incidents in Australia, New Zealand, and the United States. Inj Prev 2005;11:294-9.

2 Haworth N, Tingvall V, Kowadlo N. Review of best practice road safety initiatives in the corporate and/or business environment (No. 166). 2000. Clayton: Monash University Accident Research Centre.

3 Stuckey R, LaMontagne AD, Glass DC, et al. Estimating fatality rates in occupational light vehicle users using vehicle registration and crash data. Aust NZ J Public Health 2010;34:142-5.

4 Downs CG, Keigan M, Maycock G, et al. The safety of fleet car drivers: A review. Crowthorne House, Wokingham: Transport Research Board of the National Academies, 1999.

5 Lynn P, Lockwood CR. The accident liability of company car drivers. Crowthorne: Transport Research Laboratory, 1998.

6 Murray W, Newnam S, Watson B, et al. Evaluating and improving fleet safety in Australia. Australian Transport Safety Bureau, 2003.

7 Newnam S, Watson B, Murray W. A comparison of the factors influencing work-related drivers in a work and personal vehicle. Adelaide, Australia: Road Safety Policy, Education and Policing Conference, 2002.

8 Stuckey R, Glass DC, LaMontagne AD, et al. Risk factors for worker injury and death from occupational light vehicles crashes in New South Wales (Australia). Am J Ind Med 2010;53:931-9.

9 Newnam S, Griffin MA, Mason C. Safety in work vehicles: a multilevel study linking safety values and individual predictors to work-related driving crashes. I Appl Psychol 2008;93:632-44.

10 Newnam S, Newton C, McGregor-Lowndes M. Predicting the safety performance of volunteers: does motivation for volunteering influence driving behavior? Saf Sci 2009;47:1090-6.

11 Worksafe Australia. Workplace injury and disease recording standard. Worksafe Australia, 1990

12 Grayson G. Company cars and road safety. In: Grayson GB, ed. Behavioural research in road safety IX. Crowthorne: Transport Research Laboratory, 1999.

13 Wills $A$, Watson $B$, Biggs $H$. An exploratory investigation into safety climate and work-related driving. Work 2009;32:81-94.

14 Newnam S, Greenslade J, Newton C, et al. Safety in occupational driving: Development of a driver behavior scale for the workplace context. Appl Psychol 2011;60:576-99.

15 Adams-Guppy J, Guppy A. Speeding in relation to perceptions of risk, utility and driving style by British company car drivers. Ergonomics 1995;38:2525-35.

16 Newnam S, Griffin MA, Mason CA. Safety in work vehicles: a multi-level study linking safety values and individual predictors to work-related driving crashes. J Appl Psychol 2008;93:632-44.

17 Newnam S, Watson B. A comparison of the driving behavior betweenremunerated and volunteer drivers. Saf Sci 2011;49:369-81.

18 Zacharatos A, Barling J. High-performance work systems and occupational safety. J Appl Psychol 2005;90:77-93.

19 Griffin MA, Neal A. Perceptions of safety at work: a framework for linking safety climate to safety performance, knowledge and motivation. J Occ Health Psychol 2000;5:347-58.

20 Huselid MA. The impact of human resource practices on turnover, productivity, and corporate financial performance. Acad Manag J 1995;38:635-72.

21 Griffin MA, Talati Z. Safety leadership. In: Day D. ed.Oxford handbook of leadership and organizations. New York: Oxford University Press, 2014. In press.

22 Neal A, Griffin MA. A study of the lagged relationships among safety climate, safety motivation, safety behavior, and accidents at the individual and group levels. J Appl Psychol 2006;91:946-53.

23 Renn RW, Fedor DB. Development and field test of a feedback seeking, self-efficacy, and goal setting model of work performance. J Manag 2001;27:563-83.

24 Iversen $\mathrm{H}$, Rundmo T. Attitudes towards traffic safety, driving behavior and accident involvement among the Norwegian public. Ergonomics 2004; 47:555-72.

25 Komaki J, Heinzmann AT, Lawson L. Effect of training and feedback: component analysis of a behavioral safety program. J App/ Psychol 1980;65:261-70.

26 Zohar D. Modifying supervisory practices to improve subunit safety: $A$ leadership-based intervention model. J Appl Psychol 2002;87:153-63.

27 Zohar D, Luria G. A multilevel model of safety climate: Cross-level relationships between organization and group-level climates. J Appl Psychol 2005; 90:616-28. 


\section{Study protocol}

28 Posthuma R, Camion MC, Masimova M, et al. A high performance work practices taxomony: integrating the literature and directing future research. J Manag 2013:39:1184-220.

29 Stevenson MR, Cercarelli LR, Lee AH, et al. Estimating population-based injury prevention practices. Int J Inj Contr Saf Promot 2006;13:133-8.

30 Fleiss JL. Statistical methods for rates and proportions. 2nd ed. New York: John Wiley. 1981:38-46.
31 Bolker BM. Learning hierarchical models: advice for the rest of us. Ecol Appl 2009;19:588-92.

32 Australian Transport Council. National road safety strategy 2011-2020. Australian Transport Council, 2011.

33 Haworth N, Symonds M. Safety attitudes and behaviours in work-related driving: stage 1: analysis of crash data. Melbourne: Monash University Accident Research Centre, 2005. 